Dermatologica, 1980;161(Suppl. 1):74

\title{
Das Juvenile Melanom
}

\begin{tabular}{|l|l|l|}
\hline M. & Rupec \\
\hline A. & Kint \\
\hline W. & Horn \\
\hline
\end{tabular}

Es wurden klinische und histologische Untersuchungen an juvenilen Melanomen bei 70 Patienten (52 weibliche, 18 mannliche) durchgefuhrt. Die klinischen Befunde weichen von den bisher bekannten kaum ab. Der Tumor bestand bei 4 Patienten seit Geburt. Bei einem Patienten kam er multipel vor. $5 / 8$ Interessant ist die Tendenz der juvenilen Melanome, beim Kind im Kopfbereich, beim Erwachsenen dagegen im Extremitätenbe-reich lokalisiert zu sein. Klinisch wurde die richtige Diagnose in 35\% der Fälle gestellt.

Histologisch fanden wir auch ausserhalb der entzündlichen Infiltrate eine Proliferation der endothelialen Zellen, die manchmal morpho-logische Parallelen mit den Tumorzellen erkennen lassen. Zu ähnlichen Ergebnissen kamen, unabhängig voneinander, auch zwei weitere PathoHistologen. Die Mastzellen sind zahlreich. Im Tumorbereich besitzen sie oft einen grossen, unregelmässig gestalteten Kern und eir.en voluminosen Zelleib; wir halten sie für noch nicht ausgereifte Masto-zyten. Eine transepidermale Elimination der Tumorzellmassen wurde einmal beobachtet. Die übrigen Befunde decken sich im wesentlichen mit der schon bekannten Mikromorphologie dieser Tumoren.

Die Therapie besteht in der chirurgischen Entfernung des Tumors.

$(+)$ Eine ausführliche Veröffentlichung der Befunde ist für den Haut-arzt vorgesehen. (* > Univ.-Hautklinik Marburg/Lahn, BRD (**) Univ.-Hautklinik Gent, Belgien 